\title{
Clindamycin-induced rhabdomyolysis in the setting of mitochondrial myopathy
}

\author{
Jonathan Lowenthal*1, Jessica Kunadia ${ }^{2}$, Vivek Bose ${ }^{2}$, Jack Xu², Thomas Nahass², Isao Iwata ${ }^{2}$ \\ ${ }^{1}$ Rutgers Robert Wood Johnson Medical School, New Brunswick, United States \\ ${ }^{2}$ Department of Internal Medicine, Rutgers Robert Wood Johnson University Hospital, New Brunswick, United States
}

Received: April 5, 2019

DOI: $10.5430 /$ crim.v6n3p10
Accepted: May 26, 2019

Online Published: July 15, 2019

\begin{abstract}
We present a patient with mitochondrial myopathy who developed rhabdomyolysis following treatment with clindamycin. While clindamycin is not yet linked to drug-induced rhabdomyolysis in the literature, other drugs with mechanisms of action similar to clindamycin have been shown to damage human host mitochondria. Given this, we contend that clindamycin may also be capable of causing mitochondrial injury, and that while in otherwise healthy patients it may not produce any negative clinical outcome, it can precipitate rhabdomyolysis in certain patients whose mitochondria are already vulnerable.
\end{abstract}

Key Words: Clindamycin, Rhabdomyolysis, Drug-induced, Mitochondrial myopathy, Mitochondria, Ribosome

\section{INTRODUCTION}

Rhabdomyolysis is a well studied clinical presentation after significant damage to muscle cells occurs, causing the byproducts of degrading cells to circulate, damaging other organs that are unable to filter excessive amounts of this waste. The mitochondria in muscle cells play a central role in the production of adenosine triphosphate (ATP) and myoplasmic $\mathrm{Ca} 2+$ and when it is not functioning properly, can predispose a patient to myopathies and frequent bouts of rhabdomyolysis. ${ }^{[1]}$ Mitochondrial myopathies are rare genetic diseases and may be a risk factor for poorly studied drug-induced rhabdomyolysis. Although it is common knowledge that statins and some antibiotics may be responsible for this rare side effect, ${ }^{[2]}$ we seek to examine the effects of clindamycin as an inciting factor in rhabdomyolysis in a patient with a mitochondrial myopathy.

\section{Case presentation}

We present a 49 year-old male with mitochondrial myopathy who initially presented with non-purulent cellulitis of the left foot. The patient's medical history was significant for mitochondrial myopathy diagnosed at age 23 after presenting with Progressive External Ophthalmoplegia (PEO), ptosis, muscle wasting, muscle weakness, and ataxia. Diagnosis was confirmed with muscle biopsy showing COX deficiency, ragged red fibers, and multiple mtDNA deletions, as well as DNA sequencing that revealed a compound heterozygous mutation in the RNASEH1 gene. Following his presentation with non-purulent cellulitis, the patient was discharged on a seven day course of oral clindamycin. Blood cultures drawn during this admission were negative. On the second day of treatment, the patient began to feel profound fatigue and needed help getting to the bathroom at home. On his third day of treatment, he developed severe lower abdominal pain and returned to the hospital. On admission, he was found to have acute urinary retention. A foley catheter was placed, which not only emptied over one liter of urine, but resolved his severe abdominal pain. Urinalysis showed $2+$ blood with few red blood cells, prompting a creatinine

\footnotetext{
*Correspondence: Jonathan M. Lowenthal; Email: j11871@rwjms.rutgers.edu; Address: Rutgers Robert Wood Johnson Medical School, New Brunswick, United States.
} 
phosphokinase (CPK) to be drawn. He was found to have a CPK of 9866 units/liter (U/L) and was started on aggressive intravenous fluid resuscitation for rhabdomyolysis. Blood cultures were negative during the second admission as well. The patient reported that his fatigue started immediately after starting the clindamycin, which he had never taken before. Due to concern for an adverse medication reaction with the clindamycin, his cellulitis treatment course was switched to vancomycin for 2 more days. The patient's symptoms improved and his CPK decreased from $9888 \mathrm{U} / \mathrm{L}$ to $1393 \mathrm{U} / \mathrm{L}$ with one day of aggressive fluid treatment, and continued to downtrend until his discharge five days after admission. The etiology of his rhabdomyolysis was thought to be from the clindamycin since it had just been started several days prior to his symptoms and he had no other precipitating factors.

\section{Discussion}

Clindamycin is a bacteriostatic drug that inhibits protein synthesis by binding to the $50 \mathrm{~S}$ subunit of bacterial ribosomes. Common reported side effects of clindamycin include rash, hepatotoxicity, and diarrhea, while less common side effects include nausea, vomiting, loss of appetite, and a metallic taste. ${ }^{[3]}$ Based off our literature search, there have been no reported cases of clindamycin or other lincosamides inducing rhabdomyolysis in the clinical setting. However, there are reports of other antibiotics with similar mechanisms of action to clindamycin (ie. binding to bacterial ribosomes), targeting mitochondrial ribosome components in eukaryotic and host human cells. ${ }^{[4-10]}$ This is thought to be a result of similarities between ribosomal machinery in prokaryotic bacterial organisms and in human mitochondria, which may be explained by the theory that human mitochondria evolved from bacteria engulfed by ancient eukaryotic cells (the "endosymbiotic theory"). ${ }^{[7]}$ In healthy patients, the impact of this mitochondrial injury may be subclinical, however we hypothesize that the level of insult caused by clindamycin may be enough to precipitate a visible negative outcome in patients whose mitochondria are already damaged. While not classically associated with clindamycin, rhabdomyolysis is a well-documented possible side effect of HMG-CoA reductase inhibitors (statins), and has also been associated with certain cancer treatment drugs (tyrosine kinase inhibitors, trabectedin), disease-modifying anti-rheumatic drugs (leflunomide), antibiotics (daptomycin), and anesthetics (propofol). ${ }^{[1]}$ However, rhabdomyolysis has also been shown to occur in patients with mitochondrial disorders when stressors (such as prolonged exercise, viral illnesses, and strenuous physical activity) overtax the cells' inherently susceptible mitochondria. ${ }^{[11]}$ In our patient with mitochondrial myopathy, we believe that ribosomal damage caused by clindamycin compounded the stress on already vulnerable mitochondria, leading to the episode of acute rhabdomyolysis. While septicemia (without direct muscle infection) can also be a cause of rhabdomyolysis in the clinical setting, ${ }^{[12,13]}$ the fact that the patient's blood cultures were negative during both admissions makes it unlikely in this case. The theory that this patient's rhabdomyolysis was precipitated by his clindamycin treatment is supported by the fact that the rhabdomyolysis resolved once the clindamycin regimen was discontinued and by the lack of any adverse reaction to the vancomycin he received afterwards, as vancomycin acts by impairing bacterial cell wall synthesis and has no known effect on host mitochondria.

\section{Conclusion}

While clindamycin has not yet been linked to drug-induced rhabdomyolysis in the literature, this patient's case was strongly suggestive of a causal relationship between clindamycin and rhabdomyolysis. Given its mechanism of action and the fact that other antibiotics which share that mechanism of action have been shown to damage mitochondria in human host cells, we believe that clindamycin may also be capable of causing mitochondrial injury. While in healthy patients this level of mitochondrial insult may be insufficient to produce any negative clinical outcome, it may be sufficient in patients diagnosed with mitochondrial myopathy whose mitochondria are already inherently vulnerable to injury. Being able to identify rare side effects of commonly used medications is a difficult task. We propose that clinicians should consider including clindamycin as a potential culprit for drug-induced rhabdomyolysis in patients with underlying mitochondrial myopathy.

\section{CONFlicts OF InTEREST Disclosure}

The authors have declared no conflicts of interest.

\section{REFERENCES}

[1] Hohenegger M. Drug induced rhabdomyolysis. Curr Opin Pharmacol. 2012; 12(3): 335-9. PMid:22560920. https://doi .org/10.101 $6 / j$.coph.2012.04.002

[2] Valiyil R, Christopher-stine L. Drug-related myopathies of which the clinician should be aware. Curr Rheumatol Rep. 2010; 12(3): 21320. PMid:20425521. https://doi.org/10.1007/s11926-010 $-0104-3$

[3] Smieja M. Current indications for the use of clindamycin: A critical review. Can J Infect Dis. 1998; 9(1): 22-8. PMid:22346533. 
https://doi.org/10.1155/1998/538090

[4] Duewelhenke N, Krut O, Eysel P. Influence on mitochondria and cytotoxicity of different antibiotics administered in high concentrations on primary human osteoblasts and cell lines. Antimicrob Agents Chemother. 2007; 51(1): 54-63. PMid:17088489. https: //doi.org/10.1128/AAC.00729-05

[5] Li CH, Cheng YW, Liao PL, Yang YT, Kang JJ. Chloramphenicol causes mitochondrial stress, decreases ATP biosynthesis, induces matrix metalloproteinase-13 expression, and solid-tumor cell invasion. Toxicol Sci. 2010;116(1):140-50. PMid: 20338993. https : //doi.org/10.1093/toxsci/kfq085

[6] Mckee EE, Ferguson M, Bentley AT, Marks TA. Inhibition of mammalian mitochondrial protein synthesis by oxazolidinones.AntimicrobAgentsChemother.2006; 50(6): 2042-9. PMid:16723564. https://doi.org/10.1128/AAC.01411-05

[7] Wang X, Ryu D, Houtkooper RH, Auwerx J. Antibiotic use and abuse: a threat to mitochondria and chloroplasts with impact on research, health, and environment. Bioessays. 2015; 37(10): 1045-53. PMid: 26347282. https://doi.org/10.1002/bies. 201500071

[8] Zhang L, Ging NC, Komoda T, et al. Antibiotic susceptibility of mammalian mitochondrialtranslation. FEBSLett. 2005; 579(28): 6423-7.
PMid:16271719. https ://doi.org/10.1038/ni0901-777

[9] Barnhill AE, Brewer MT, Carlson SA. Adverse effects of antimicrobials via predictable or idiosyncratic inhibition of host mitochondrial components. Antimicrob Agents Chemother. 2012; 56(8): 4046-51. PMid:22615289. https://doi.org/10.1128/AAC.00678-12

[10] Hong S, Harris KA, Fanning KD, et al. Evidence That Antibiotics Bind to Human Mitochondrial Ribosomal RNA Has Implications for Aminoglycoside Toxicity. J Biol Chem. 2015; 290(31): $19273-$ 86. PMid: 26060252. https://doi.org/10.1074/jbc.M115.6 55092

[11] Scalco RS, Gardiner AR, Pitceathly RD, et al. Rhabdomyolysis: a genetic perspective. Orphanet J Rare Dis. 2015; 10: 51. PMid:25929793.

[12] Posner MR, Caudill MA, Brass R, Ellis E. Legionnaires' disease associated with rhabdomyolysis and myoglobinuria.Arch Intern Med 1980; 140(6): 848. PMid:7387285. https://doi.org/10.1001/ archinte.1980.00330180122038

[13] Naschitz JE, Yeshurun D, Shagrawi I. Rhabdomyolysis in pneumococcal sepsis. Am J Med. 1989; 87(4): 479-80. https : //doi .org/ $10.1016 / \mathrm{S} 0002-9343(89) 80842-3$ 